\title{
In situ measurements of the oblique incidence sound absorption coefficient for finite sized absorbers
}

Ottink, Marco; Brunskog, Jonas; Jeong, Cheol-Ho; Fernandez Grande, Efren; Trojgaard, Per; Tiana Roig, Elisabet

Published in:

Journal of the Acoustical Society of America

Link to article, DOI:

$10.1121 / 1.4938225$

Publication date:

2016

Document Version

Publisher's PDF, also known as Version of record

Link back to DTU Orbit

Citation (APA):

Ottink, M., Brunskog, J., Jeong, C-H., Fernandez Grande, E., Trojgaard, P., \& Tiana Roig, E. (2016). In situ measurements of the oblique incidence sound absorption coefficient for finite sized absorbers. Journal of the Acoustical Society of America, 139(1), 41-52. https://doi.org/10.1121/1.4938225

\section{General rights}

Copyright and moral rights for the publications made accessible in the public portal are retained by the authors and/or other copyright owners and it is a condition of accessing publications that users recognise and abide by the legal requirements associated with these rights.

- Users may download and print one copy of any publication from the public portal for the purpose of private study or research.

- You may not further distribute the material or use it for any profit-making activity or commercial gain

- You may freely distribute the URL identifying the publication in the public portal 


\title{
In situ measurements of the oblique incidence sound absorption coefficient for finite sized absorbers
}

\author{
Marco Ottink ${ }^{\text {a) }}$ \\ Müller-BBM GmbH, Robert-Koch-Strasse 11, Planegg, 82152, Germany \\ Jonas Brunskog, Cheol-Ho Jeong, and Efren Fernandez-Grande \\ Acoustic Technology, DTU Electrical Engineering, Technical University of Denmark, Ørsteds Plads 352, \\ DK-2800 Kongens Lyngby, Denmark \\ Per Trojgaard \\ Lloyds Register Consulting—Engineering Dynamics, Strandvejen 104A, DK-2900 Hellerup, Denmark \\ Elisabet Tiana-Roig \\ Brüel and Kjar Sound and Vibration Measurement A/S, Skodsborgvej 307, Narum, DK-2850, Denmark
}

(Received 31 January 2015; revised 2 November 2015; accepted 4 December 2015; published online 5 January 2016)

\begin{abstract}
Absorption coefficients are mostly measured in reverberation rooms or with impedance tubes. Since these methods are only suitable for measuring the random incidence and the normal incidence absorption coefficient, there exists an increasing need for absorption coefficient measurement of finite absorbers at oblique incidence in situ. Due to the edge diffraction effect, oblique incidence methods considering an infinite sample fail to measure the absorption coefficient at large incidence angles of finite samples. This paper aims for the development of a measurement method that accounts for the finiteness of the absorber. A sound field model, which accounts for scattering from the finite absorber edges, assuming plane wave incidence is derived. A significant influence of the finiteness on the radiation impedance and the corresponding absorption coefficient is found. A finite surface method, which combines microphone array measurements over a finite sample with the sound field model in an inverse manner, is proposed. Besides, a temporal subtraction method, a microphone array method, impedance tube measurements, and an equivalent fluid model are used for validation. The finite surface method gives promising agreement with theory, especially at near grazing incidence. Thus, the finite surface method is proposed for further measurements at large incidence angles. (0) 2016 Acoustical Society of America. [http://dx.doi.org/10.1121/1.4938225]
\end{abstract}

[MV]

Pages: 41-52

\section{INTRODUCTION}

Sound absorption coefficient measurements are mostly carried out with impedance tubes following ISO $10534^{1}$ (for normal incidence), or in reverberation chambers by the chamber method according to ISO $354^{2}$ (for random incidence). It is widely accepted that the absorption coefficient measured by the chamber method can exceed unity for finite sized samples due to the so-called edge diffraction effect. This behavior, also known as the size effect, is well described by Thomasson.,

Since the room method is based on reverberation time measurements with the assumption of a diffuse sound field, it is just suitable to describe absorber properties for random sound incidence. Nevertheless, the absorption coefficient at an incidence angle of 45 degrees is found to be a good estimate of the random incidence condition. ${ }^{5}$ Besides this, there exists an increasing need to better describe absorber properties depending on the angle of incidence. The angle dependent absorption coefficient could, e.g., be used as an input parameter in room acoustic simulations. Analytical or theoretical procedures to describe angle dependent absorbent

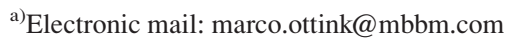

properties are available, but experimental validations are required.

In the early 1950s, Ingard and Bolt ${ }^{6}$ presented a free field method based on sound pressure magnitude and phase measurements of the incident and reflected sound wave at an absorbing material. Their results showed that it is difficult to compute the absorption coefficient with increasing incidence angle. To improve the separation of the incident and reflected sound waves, Mommertz presented a subtraction method based on impulse response measurements in free field above the absorber. ${ }^{7}$ By means of post processing, the measurement was improved due to the separation of the direct and reflected impulse responses. Robinson and Xiang ${ }^{8}$ improved this method further by oversampling the measured responses. They concluded that even with a careful post processing of the measured impulse responses, it is difficult to get reliable results at large incidence angles. A slightly different approach was presented by Hirosawa et al., ${ }^{9}$ using three different in situ methods based on pressure-pressure, pressure-velocity, and velocity-velocity measurements on a vertical line at two positions above the absorbing material. As a key observation, they showed that for finite absorber samples a correction for the non-planar wave field has to be applied. Furthermore, Yori and Moeser ${ }^{10}$ presented an 
alternative measurement approach based on a microphone arrangement to measure sound pressure distribution on a plane from which they calculated the complex pressure reflection coefficient by using a spatial Fourier transform. A literature review of in situ acoustic measurement techniques was presented by Brandao et al. ${ }^{11}$

All these in situ methods have the disadvantage that the theory is based on an infinite sample size, while the measurements were carried out with a finite sample. The effect of the finite sample size of the absorber has been studied and explained by Tomasson, ${ }^{3,4}$ using a variational approach. Mechel $^{12}$ has investigated this effect based on Thomasson's model. It is evident that the finiteness of the absorber has a sufficient influence on the measured absorption coefficient for large incidence angles, which could explain why in situ methods have difficulties in getting reliable measurement results for large incidence angles. The goal of the present study is to overcome these difficulties by taking the finiteness of the absorber into account. Therefore, Thomasson's theory is extended to predict the sound field, based on plane wave incidence, in front of a finite sized absorber placed on a rigid infinite baffle, which is used in an inverse measurement approach to estimate the impedance of the absorber.

To validate the theory, three in situ methods: the temporal subtraction method, the microphone array method, and the finite surface method are used. As reported by Brandao et al., ${ }^{11}$ measurements in an anechoic chamber can be used to mimic the mounting conditions of in situ measurements. Hence, in situ measurements in this paper are performed in an anechoic chamber.

\section{THEORY}

Throughout this paper, the time convention $e^{\mathrm{j}^{\omega t}}$ is used, where $\omega=2 \pi f$ is the angular frequency, $f$ the frequency, and $t$ is the time. For the surface impedance, the surface impedance $Z_{A}$, normalized by $\rho c$, is used, where $c$ is the speed of sound and $\rho$ is the density of air.

\section{A. Simplified plane wave reflections of an infinite absorber surface}

In this section, the calculations of absorber properties using a simplified plane wave reflection assumption are presented. More details are found in Refs. 12 and 13. We first consider an infinite absorber sample (placed on a rigid infinite backing). By assuming simple plane wave reflections from the infinite absorber, the absorption coefficient $\alpha_{\infty}$ is calculated as

$$
\alpha_{\infty}(\theta)=1-|R(\theta)|^{2}
$$

where $\theta$ is the incidence angle with respect to the perpendicular of the material plane and $R(\theta)$ is the reflection factor, which for plane waves is defined as the complex pressure amplitude ratio of the incident to reflected plane waves, $\hat{p}_{i}$ and $\hat{p}_{r}$, respectively, i.e., $R(\theta)=\hat{p}_{r} / \hat{p}_{i}{ }^{9-15}$ The corresponding surface impedance of the absorber is calculated from the reflection factor as

$$
Z_{A}=\frac{1}{\cos (\theta)} \frac{1+R(\theta)}{1-R(\theta)} .
$$

Combining Eq. (1) with Eq. (2), the absorption coefficient of an infinite absorber is calculated as

$$
\alpha_{\infty}(\theta)=1-\left|\frac{Z_{A}-\frac{1}{\cos (\theta)}}{\mid Z_{A}+\frac{1}{\cos (\theta)}}\right|^{2}=\frac{4 \Re\left\{Z_{A}\right\} \frac{1}{\cos \theta}}{\left|Z_{A}+\frac{1}{\cos \theta}\right|^{2}} .
$$

It should be noticed that to consider a spherical wave reflection in an infinite impedance boundary, the exact solution is more complicated than what can be described with a plane wave reflection factor; see, e.g., Ref. 16. Furthermore, the assumption of plane reflecting waves associated with the infinite size of the absorber does not account for scattering effects that occur due to the finiteness of the absorber. To consider this, a more detailed sound field model is needed.

\section{B. Sound field model considering scattering from a finite absorber}

In this section, a more detailed sound field model is presented. The model of the sound field is based on the methods described by Thomason ${ }^{3,4}$ and Morse and Ingard. ${ }^{17}$ Further details about the variational formulation and the finiteness issues are found in Ref. 14.

In the proposed sound field model, an incident plane wave and a specular plane wave, as shown in Fig. 1, are considered.

An absorber of arbitrary shape with surface area $S$ ( $S=a b$ for a rectangular absorber) and admittance $\beta$ $=1 / Z_{A}$ is placed on a surface with admittance $\beta_{0}$. Here, the normalized surface impedance $Z_{A}$ for finite sized absorbers generally depends on the angle of incidence, as shown in Ref. 13. The sound pressures of the positive and negative traveling waves (incident and specularly reflected plane wave) are given by

$$
\begin{aligned}
& p_{i}(x, y, z)=\hat{p}_{i} e^{-\mathrm{j}\left(k_{x} x+k_{y} y+k_{z} z\right)}, \\
& p_{r}(x, y, z)=\hat{p}_{r} e^{-\mathrm{j}\left(k_{x} x+k_{y} y-k_{z} z\right)},
\end{aligned}
$$

where $\hat{p}_{i}$ is the complex amplitude of the incident plane wave and $\hat{p}_{r}$ is the complex amplitude of the reflected plane

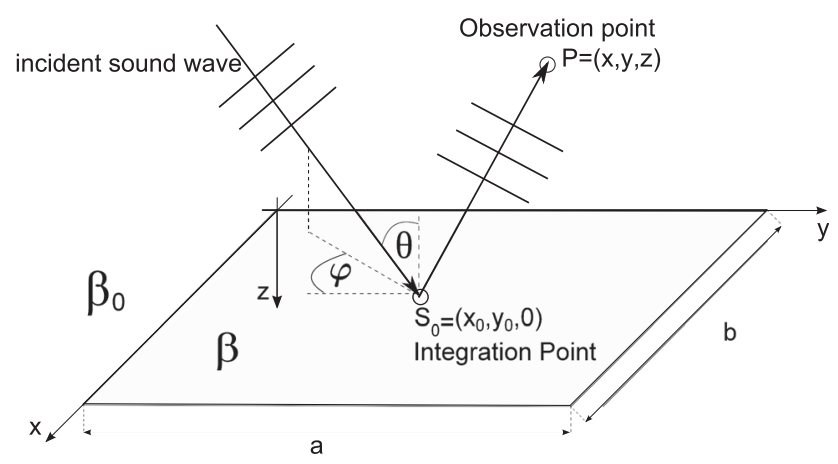

FIG. 1. Sketch of the sound field model, including scattering from a finite surface. 
wave. The influences of scattering from the surrounding baffle and from the absorber surface are considered separately. Thus, the pressure amplitudes for the reflected and incident plane waves are considered to be equal, corresponding to a perfect reflection. The wave numbers $k_{x}, k_{y}$, and $k_{z}$ can be expressed in terms of the incident angle $\theta$ and the azimuth angle $\varphi$ as $k_{x}=k \sin \theta \cos \varphi, \quad k_{y}=k \sin \theta \sin \varphi$, $k_{z}=k \cos \theta$, where $k=\omega / c$ is the wave number in air. To specify the resulting sound pressure at a point $P \in(x, y, z)$, resulting from an incident plane wave, scattering from the surface is taken into account. The sound pressure at point $P$ is described as an integral equation, ${ }^{17}$

$$
\begin{aligned}
p(x, y, z)= & \hat{p}_{i}\left(e^{-\mathrm{j}\left(k_{x} x+k_{y} y+k_{z} z\right)}+e^{-\mathrm{j}\left(k_{x} x+k_{y} y-k_{z} z\right)}\right) \\
& -\mathrm{j} k \Delta \beta \int_{S} G\left(x, y, z \mid x_{0}, y_{0}, 0\right) p\left(x_{0}, y_{0}, 0\right) \mathrm{d} S_{0},
\end{aligned}
$$

where $\Delta \beta=\beta-\beta_{0}$ is the change of admittance between the absorber and the surrounding baffle, $p\left(x_{0}, y_{0}, 0\right)$ is the pressure function on the absorber surface at $z=0, \int_{S} \mathrm{~d} S_{0}$ is the integral over the arbitrary material surface and $G\left(x, y, z \mid x_{0}, y_{0}, 0\right)$ is the half space Green's function between the respective integration point $S_{0} \in\left(x_{0}, y_{0}, 0\right)$ and the observation point $P$ :

$$
G\left(x, y, z \mid x_{0}, y_{0}, 0\right)=\frac{e^{-\mathrm{j} k d}}{2 \pi d} .
$$

The distance $d$ between the observation point $P$ and the surface point $S_{0}$ is given by $d=\sqrt{\left(x-x_{0}\right)^{2}+\left(y-y_{0}\right)^{2}+z^{2}}$. Equation (5) holds for locally reacting absorbers and also for non-locally reacting absorbers. ${ }^{3,4,12}$ This can be seen if studying Ref. 18, where the corresponding theory is applied to a plate in bending, which is non-locally reacting. In the present case, when expressing the admittance as an differential operator $\mathcal{B}\{p\}$, this will be converted into an ordinary linear admittance if the pressure field is a projected plane wave $\mathcal{B}\left\{\hat{p} e^{-\mathrm{j}\left(k_{x} x+k_{y} y\right)}\right\}=\beta\left(k_{x} k_{y}\right) \hat{p} e^{-\mathrm{j}\left(k_{x} x+k_{y} y\right)}$. This will lead to an angle dependent impedance $Z_{A}(\theta, \varphi)$, which clearly is not locally reacting. Thus, the theory is not relying on the locally reacting assumption.

\section{Variational formulation of the integral equation}

The unknown sound pressure $p$ in the integral equation, Eq. (5), cannot be solved explicitly. Thus, a variational principle described in Ref. 17 is employed, which was also used in Refs. 3,4,17, and 18-20. The variational formulation is found by multiplying with an adjoint pressure field and then integrating as

$$
\begin{aligned}
& \int_{S} p(x, y, 0) p_{a}(x, y, 0) \mathrm{d} S \\
& =\int_{S} 2 p_{i}(x, y) p_{a}(x, y) \mathrm{d} S \\
& \quad-\mathrm{j} k \Delta \beta \int_{S} \int_{S} G\left(x, y \mid x_{0}, y_{0}\right) p\left(x_{0}, y_{0}\right) p_{a}(x, y) \mathrm{d} S_{0} \mathrm{~d} S,
\end{aligned}
$$

where $p_{a}(x, y, 0)$ is the adjoint sound pressure of the negative traveling wave on the absorber. The variational functional $V(p)$ is defined to be

$$
V(p)=2 \hat{p}_{i} \int_{S} e^{\mathrm{j}\left(k_{x} x+k_{y} y\right)} p(x, y) \mathrm{d} S
$$

which is related to the sound power. Combining Eqs. (7) and (8) leads to the final form of the variational functional of the integral equation

$$
\begin{aligned}
V\left(p, p_{a}\right)= & \hat{p}_{i} \int_{S} H(x, y) \mathrm{d} S-\int_{S} p(x, y) p_{a}(x, y) \mathrm{d} S \\
& -\mathrm{j} k \Delta \beta \int_{S} \int_{S} G\left(x, y \mid x_{0}, y_{0}\right) p\left(x_{0}, y_{0}\right) p_{a}(x, y) \mathrm{d} S_{0} \mathrm{~d} S,
\end{aligned}
$$

with

$$
H(x, y)=e^{\mathrm{j}\left(k_{x} x+k_{y} y\right)} p(x, y)+e^{-\mathrm{j}\left(k_{x} x+k_{y} y\right)} p_{a}(x, y) .
$$

This functional is symmetric with respect to $p$ and $p_{a}$, and will be optimized in order to find a variational solution.

\section{Solution of the variational equation}

To find the solution to the variational problem, the traveling waves on the absorber surface $p(x, y, 0)$ and $p_{a}(x, y, 0)$ are assumed in terms of a positive and a negative traveling wave as

$$
\begin{aligned}
& p(x, y)=\hat{p}_{v} e^{-\mathrm{j}\left(k_{x} x+k_{y} y\right)}, \\
& p_{a}(x, y)=\hat{p}_{v} e^{\mathrm{j}\left(k_{x} x+k_{y} y\right)},
\end{aligned}
$$

where $\hat{p}_{v}$ is the unknown complex amplitude of the pressure at the integration point $S_{0}$. Inserting Eqs. (11) and (12) into Eq. (9) yields

$$
\begin{aligned}
V\left(\hat{p}_{v}\right)= & 4 \hat{p}_{i} \hat{p}_{v} S-\hat{p}_{v}^{2} S-\mathrm{j} k \Delta \beta \int_{S} \int_{S} G\left(x, y \mid x_{0}, y_{0}\right) \\
& \times \hat{p}_{v}^{2} e^{\mathrm{j}\left(k_{x}\left(x-x_{0}\right)+k_{y}\left(y-y_{0}\right)\right)} \mathrm{d} S_{0} \mathrm{~d} S,
\end{aligned}
$$

and the optimal of the functional is found by letting the derivative with respect to $\hat{p}_{v}$ be zero,

$$
\begin{aligned}
\frac{\partial V\left(\hat{p}_{v}\right)}{\partial \hat{p}_{v}}= & 4 \hat{p}_{i} S-2 \hat{p}_{v} S-\mathrm{j} k \Delta \beta \int_{S} \int_{S} G\left(x, y \mid x_{0}, y_{0}\right) 2 \hat{p}_{v} \\
& \times e^{\mathrm{j}\left(k_{x}\left(x-x_{0}\right)+k_{y}\left(y-y_{0}\right)\right)} \mathrm{d} S_{0} \mathrm{~d} S=0 .
\end{aligned}
$$

The integral can be identified as the normalized specific radiation impedance, ${ }^{3}$

$$
Z_{F}=-\frac{\mathrm{j} k}{S} \int_{S} \int_{S} e^{\mathrm{j}\left(k_{x}\left(x-x_{0}\right)+k_{y}\left(y-y_{0}\right)\right)} G\left(x, y \mid x_{0}, y_{0}\right) \mathrm{d} S_{0} \mathrm{~d} S .
$$

The radiation impedance depends on the incidence angle $\theta$ and the azimuth angle $\varphi$. Solving Eq. (14) for $\hat{p}_{v}$ results in 


$$
\hat{p}_{v}=\frac{2 \hat{p}_{i}}{1+\Delta \beta Z_{F}}=\frac{2 \hat{p}_{i} Z_{A}}{Z_{A}+Z_{F}} .
$$

For large Helmholtz numbers, $k e$, the surface impedance $\mathrm{Z}_{F}$ approaches $1 / \cos (\theta)$. The factor $1 / \cos \theta \operatorname{can}$ also be seen in Eq. (2) for an infinite absorber. Thus, for large Helmholtz numbers, the simplified plane wave reflection of an infinite absorber surface, shown in Sec. II A, gives accurate results. For small Helmholtz numbers, the simplified plane wave reflection of an infinite absorber surface is just an approximation for small incidence angles. This does not hold anymore at near grazing incidence.

In this study, it is assumed that the finite absorber is surrounded by a rigid infinite baffle $\left(\beta_{0}=0\right)$. Therefore, $Z_{A}$ represents the surface impedance of the absorbing material. By substituting the variational solution from Eq. (16), Eq. (5) is approximated as

$$
\begin{aligned}
p(x, y, z)= & \hat{p}_{i}\left(e^{-\mathrm{j}\left(k_{x} x+k_{y} y+k_{z} z\right)}+e^{-\mathrm{j}\left(k_{x} x+k_{y} y-k_{z} z\right)}\right) \\
& -\hat{p}_{i} \frac{2 \mathrm{j} k F\left(x, y, z \mid x_{0}, y_{0}\right)}{Z_{A}+Z_{F}}
\end{aligned}
$$

where

$$
F\left(x, y, z \mid x_{0}, y_{0}\right)=\int_{S} G\left(x, y, z \mid x_{0}, y_{0}, 0\right) e^{-\mathrm{j}\left(k_{x} x_{0}+k_{y} y_{0}\right)} \mathrm{d} S_{0} .
$$

In case of observing the sound pressure on the absorber surface at $z=0$, Eq. (17) is rewritten as

$$
p(x, y)=2 \hat{p}_{i}\left(e^{-\mathrm{j}\left(k_{x} x+k_{y} y\right)}-\frac{\mathrm{j} k F\left(x, y, 0 \mid x_{0}, y_{0}\right)}{Z_{A}+Z_{F}}\right) .
$$

In Sec. VI A, numerical results of the sound pressure on the absorber surface can be found.

\section{Computation of the radiation impedance}

The radiation impedance, Eq. (15), can be fully determined by the size and geometry of the absorber, as shown in Refs. 3 and 4. In the experiments presented in this paper, a rectangular absorber with the side length $e=a=b$ is used. However, the radiation impedance for a rectangular absorber in the form given in Eq. (15) is not well suited for numerical integration. Thus, a numerically more convenient expression with just two integrals, following the procedure described by Brunskog, ${ }^{18}$ based on a variable substitution, ${ }^{21}$ is given by

$$
\begin{aligned}
Z_{F}= & -\frac{\mathrm{j} k}{2 \pi S} \int_{0}^{a} \int_{0}^{b} 4 \cos \left(k_{x} \kappa\right) \cos \left(k_{y} \tau\right) \\
& \times \frac{e^{-\mathrm{j} k \sqrt{\kappa^{2}+\tau^{2}}}}{\sqrt{\kappa^{2}+\tau^{2}}}(a-\kappa)(b-\tau) \mathrm{d} \kappa \mathrm{d} \tau .
\end{aligned}
$$

This expression is easy to implement in numerical quadrature routines, but it still requires a high computational cost. However, Thomasson ${ }^{4}$ developed a high frequency approximation of the radiation impedance. Davy et $a l^{22}$ recently established an updated simplified expression, which is an alternative approximation of Thomasson's high frequency approximation. In this paper, numerical integration of Eq. (20) is used and the approximate expression is avoided.

\section{Computation of the absorption coefficient}

The ordinary definition of the absorption coefficient $\alpha(\theta)$ is defined in terms of dividing the absorbed power, for the finite absorber being ${ }^{3}$

$$
P_{\text {abs }}=\frac{S\left|p_{i}\right|^{2}}{2 \rho c} \frac{\Re\left\{Z_{A}\right\}}{\left|Z_{A}+Z_{F}\right|^{2}},
$$

by the projected incident power being

$$
P_{\text {in,proj }}=\frac{S\left|p_{i}\right|^{2}}{2 \rho c} \cos \theta
$$

from which follows

$$
\alpha(\theta)=\frac{P_{\text {abs }}}{P_{\text {in,proj }}}=\frac{4 \Re\left\{Z_{A}\right\} \frac{1}{\cos \theta} .}{\left|Z_{A}+Z_{F}\right|^{2}} .
$$

It is well known that this absorption coefficient can exceed unity due to the dependency on the absorber area. ${ }^{23}$ For an infinite absorber sample, $Z_{F} \rightarrow 1 / \cos \theta$, Eq. (23) is identical to the findings from Sec. II A.

Besides this ordinary definition, Thomasson provides an alternative absorption coefficient $\alpha^{\prime}(\theta)$ in terms of dividing the absorbed power of a finite absorber by the maximum available power

$$
P_{\max }=\frac{S\left|p_{i}\right|^{2}}{2 \rho c} \frac{1}{\Re\left\{Z_{F}\right\}},
$$

for each plane wave, leading to

$$
\alpha^{\prime}(\theta)=\frac{P_{\mathrm{abs}}}{P_{\max }}=\frac{4 \Re\left\{Z_{A}\right\} \Re\left\{Z_{F}\right\}}{\left|Z_{A}+Z_{F}\right|^{2}} .
$$

Due to the dependency on the maximum available power, this alternative absorption coefficient cannot exceed unity for small specimens. It is thus less dependent on the absorber area, but still contains effects of the size and shape of the absorber. ${ }^{3}$ Therefore, the alternative absorption coefficient might be well suitable for room acoustic computer simulations. Moreover, it will have numerical numbers more comparable with the ordinary absorption coefficient for finite sized absorbers, Eq. (3), making it suitable for the comparisons in Sec. VI.

Hence, Thomasson's alternative absorption coefficient in Eq. (25) is used when comparing with the finite surface method, and Eq. (1) is used for the other two methods, assuming an infinite absorber sample.

\section{IN SITU MEASUREMENT METHODS}

Three in situ methods to measure absorber properties are introduced in the following. Besides the well accepted, 
temporal subtraction method, ${ }^{7,8}$ an alternative method based on microphone array measurements, referred to here as the microphone array method, is presented.

Both methods are described and detailed in Secs. III A and III B. Using these two methods, the absorption coefficient $\alpha(\theta)$ is calculated by Eq. (1) by assuming an infinite absorber sample. Finally, a finite surface method that takes into account the finiteness of the absorber using an inverse technique is proposed. This method is described in detail in Sec. IIIC. The finite surface method estimates the surface impedance $Z_{A}$. From the surface impedance, the absorption coefficient $\alpha^{\prime}(\theta)$ is calculated by Eq. (25), so that comparison can be made with the other two methods.

\section{A. Temporal subtraction method}

The temporal subtraction method is based on impulse response measurements at a certain distance above the surface under test in anechoic conditions by using a single microphone. For post processing, a subtraction method as in Refs. 7 and 8 is used. Therefore, another impulse response is measured without the absorbing material by keeping the loudspeaker-microphone position the same. The absorption coefficient of the test surface is calculated from the postprocessed direct and reflected part of the impulse responses by taking the geometrical spreading between the amplitudes of the respective parts into account, following ISO $13472-1 .{ }^{24}$

This temporal subtraction method has the advantage that it is simple to implement, but it has the disadvantage that no compensation for the finiteness of the absorber is made.

\section{B. Microphone array method}

The microphone array method is based on acoustic holography with an array of microphones in two parallel planes above the absorber sample. In acoustic holography, microphone array measurements in a two-dimensional space (hologram plane) are used to reconstruct a sound field in a three-dimensional space by accounting for the propagation of the sound waves between the measurement and reconstruction points. ${ }^{25-29} \mathrm{~A}$ well-suited method is the so-called statistically optimized near-field acoustic holography (SONAH). ${ }^{26,27}$ Figure 2 shows a sketch of the microphone array setup. By using SONAH, the sound field in two measurement planes, $z_{h 1}$ and $z_{h 2}$, is expressed as a decomposition of elementary waves, each having an associated coefficient $\left(c_{r}\right.$ and $\left.c_{i}\right) .{ }^{27}$ In principle, the method is valid to describe any arbitrary waveform based on a plane wave expansion. However, in this study, a plane wave incidence is implicitly assumed, as shown in Sec. II B. In a matrix form, the sound field in the two measurement planes can be expressed as

$$
\left[\begin{array}{l}
\mathbf{p}\left(\mathbf{r}_{h 1}\right) \\
\mathbf{p}\left(\mathbf{r}_{h 2}\right)
\end{array}\right]=\mathbf{A}\left[\begin{array}{l}
\mathbf{c}_{r} \\
\mathbf{c}_{i}
\end{array}\right]=\left[\begin{array}{ll}
\mathbf{A}_{r 1} & \mathbf{X}_{i 1} \\
\mathbf{A}_{r 2} & \mathbf{X}_{i 2}
\end{array}\right]\left[\begin{array}{l}
\mathbf{c}_{r} \\
\mathbf{c}_{i}
\end{array}\right]
$$

where $\mathbf{A}$ is the matrix containing the elementary wave functions, $\mathbf{p}\left(\mathbf{r}_{h 1}\right)$ and $\mathbf{p}\left(\mathbf{r}_{h 2}\right)$ are the vectors describing the sound pressure in the two hologram planes $\left(z_{h_{1}}\right.$ and $\left.z_{h_{2}}\right)$, and $\mathbf{c}_{r}$ and

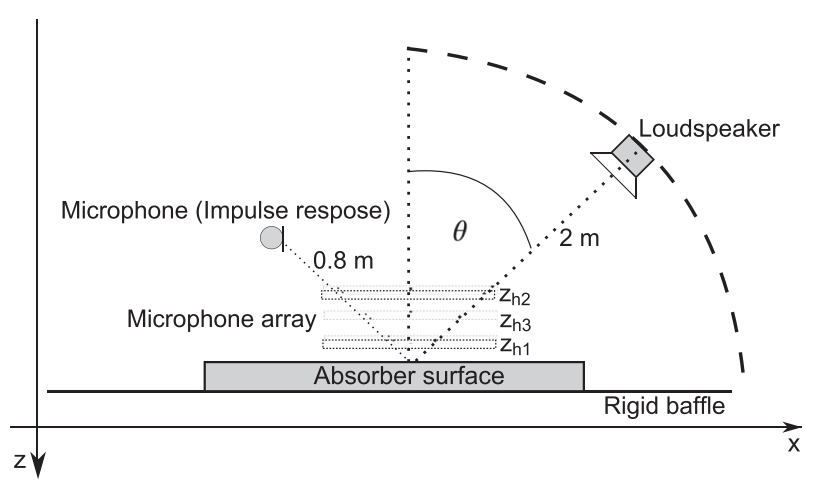

FIG. 2. Sketch of the different measurement setups. The single microphone is used for the temporal subtraction method, while the microphone array is used for the microphone array method, and the finite surface method.

$\mathbf{c}_{i}$ are the corresponding coefficients of the incident and the reflected waves (here, reflected from the absorber surface) ${ }^{26,27} \mathbf{A}_{r 1}$ and $\mathbf{X}_{i 1}$ are the matrices of the reflected (positive going) and incident (negative going) elementary wave functions in the first measurement plane $\left(z_{h 1}\right)$, and $\mathbf{A}_{r 2}$ and $\mathbf{X}_{i 2}$ are the matrices of the reflected and incident elementary wave functions, respectively, in the second measurement plane $\left(z_{h 2}\right)$. In this study, the matrices contain the respective elementary wave functions of each microphone position in the respective hologram plane. Each elementary wave function for each microphone position can be expressed as

$$
\begin{aligned}
A_{r 1, n} & =e^{-\mathrm{j}\left(k_{x} x_{n}+k_{y} y_{n}-k_{z}\left(z_{n 1}-z_{0}\right)\right)}, \\
A_{r 2, n} & =e^{-\mathrm{j}\left(k_{x} x_{n}+k_{y} y_{n}-k_{z}\left(z_{n 2}-z_{0}\right)\right)}, \\
X_{i 1, n} & =e^{-\mathrm{j}\left(k_{x} x_{n}+k_{y} y_{n}+k_{z}\left(z_{n 1}-z_{x}\right)\right)}, \\
X_{i 2, n} & =e^{-\mathrm{j}\left(k_{x} x_{n}+k_{y} y_{n}+k_{z}\left(z_{n 2}-z_{x}\right)\right)},
\end{aligned}
$$

where $z_{0}$ and $z_{x}$ are the virtual planes in which the elementary waves are scaled and $n$ indicates the specific microphone of the array. ${ }^{26}$ The coefficients are solved in a leastsquares sense using Tikhonov regularization. ${ }^{30,31}$ Making use of the estimated coefficients, the sound pressure due to the incident and reflected waves in the reconstruction plane $z_{s}$ can be calculated as

$$
\begin{aligned}
& \mathbf{p}_{r}\left(\mathbf{r}_{s}\right)=\mathbf{B}_{r} \mathbf{c}_{r}, \\
& \mathbf{p}_{i}\left(\mathbf{r}_{s}\right)=\mathbf{B}_{i} \mathbf{c}_{i},
\end{aligned}
$$

where the vectors $\mathbf{p}_{r}\left(\mathbf{r}_{s}\right)$ and $\mathbf{p}_{i}\left(\mathbf{r}_{s}\right)$ represent the pressures due to the reflected and the incident waves in the reconstruction plane, respectively, and the matrices $\mathbf{B}_{r}$ and $\mathbf{B}_{i}$ represent the reflected and the incident waves in the reconstruction plane, respectively. Each element of these matrices, depending on the position of the $n$th reconstruction point is, respectively, given by

$$
\begin{aligned}
& B_{r, n}=e^{-\mathrm{j}\left(k_{x} x_{n}+k_{y} y_{n}-k_{z}\left(z_{s}-z_{x}\right)\right)}, \\
& B_{i, n}=e^{-\mathrm{j}\left(k_{x} x_{n}+k_{y} y_{n}+k_{z}\left(z_{s}-z_{0}\right)\right)} .
\end{aligned}
$$

Making use of the incident and the reflected sound pressure close to the absorber, the generalized definition of the reflection factor, see Eq. (2) of Ref. 11, for the $n$th reconstruction point is used to form an average reflection factor, 


$$
\left|\left\langle R_{n}\right\rangle\right|=\left\|\mathbf{p}_{\mathbf{r}, \mathbf{n}}\left(\mathbf{r}_{\mathbf{s}}\right) \oslash \mathbf{p}_{\mathbf{i}, \mathbf{n}}\left(\mathbf{r}_{\mathbf{s}}\right)\right\|_{2},
$$

where \|\|$_{2}$ is the 2-norm, " \langle\rangle " indicates a spatial average and " $\oslash$ " represents a pointwise division inherent to plane wave reflection; therefore, an apparent plane wave reflection factor is estimated via Eq. (30), where the 2-norm acts as a spatial average.

The microphone array method used is a patch method that circumvents replicated aperture errors associated with the discrete Fourier transform (DFT). Fourier based methods, such as the Tamura method, ${ }^{32,33}$ assume spatial periodicity of the sound field. Consequently, the present microphone array method is more accurate. ${ }^{26}$ Nevertheless, the microphone array method has the advantage that it is based on the average results computed from several microphones, but it also has the disadvantage that no compensation for the finiteness of the absorber is made since $\alpha_{\infty}$ is calculated using Eq. (1), although it would also be possible to use Eq. (25).

\section{Finite surface method}

Besides the temporal subtraction method and the microphone array method that are mainly used for comparison, we propose a method called the finite surface method. This method considers the influence of the absorber finiteness, which is the main focus of this study. To estimate the surface impedance for oblique sound incidence and the corresponding absorption coefficient, the finite surface method combines the sound field model, described in Sec. II B with microphone array measurements in an inverse manner. For calculations with the sound field model, Eq. (17), the position of each microphone and the total sound pressure at each frequency and microphone position should be known. The radiation impedance $Z_{F}$ is known from Eq. (20). The unknowns in Eq. (17) are the amplitude of the incident sound pressure $\hat{p}_{i}$ and the surface impedance $Z_{A}$ of the test material. The incident sound pressure amplitude at the surface can be estimated from the microphone array method, as shown in Eq. (28) and Ref. 28.

Since the measurements are performed with $N$ microphones at discrete positions, the sound pressure amplitude $\hat{p}_{i}$ can be estimated by minimizing the least-squares error as

$$
\begin{aligned}
E_{L S}\left(\hat{p}_{i}\right)= & \frac{1}{S} \int_{S} \mid \hat{p}_{i} e^{-\mathrm{j}\left(k_{x} x_{0, n}+k_{y} y_{0, n}+k_{z} z_{0, n}\right)} \\
& -\left.p_{i}\left(x_{0, n}, y_{0, n}, z_{0, n}\right)\right|^{2} \mathrm{~d} S \\
& \simeq \sum_{n=1}^{N}\left|\hat{p}_{i} e^{-\mathrm{j}\left(k_{x} x_{0, n}+k_{y} y_{0, n}+k_{z} z_{0, n}\right)}-p_{i}\left(x_{0, n}, y_{0, n}, z_{0, n}\right)\right|^{2}
\end{aligned}
$$

where $p_{i}\left(x_{0, n}, y_{0, n}, z_{0, n}\right)$ is the estimated incident sound pressure at position $n$ obtained with SONAH and $\hat{p}_{i} e^{-\mathrm{j}\left(k_{x} x_{0, n}+k_{y} y_{0, n}+k_{z} z_{0, n}\right)}$ is the incident sound pressure at position $n$, where $\hat{p}_{i}$ is the incident pressure amplitude.

By using the estimated incident sound pressure amplitude from Eq. (31), the surface impedance is determined by minimizing the least-square error

$$
\begin{aligned}
E_{L S}\left(Z_{A}\right) & =\frac{1}{S} \int_{S}\left|p_{f w}\left(x, y, z \mid Z_{A}\right)-p_{t}(x, y, z)\right|^{2} \mathrm{~d} S \\
& \simeq \sum_{n=1}^{N}\left|p_{f w, n}\left(x, y, z \mid Z_{A}\right)-p_{t, n}(x, y, z)\right|^{2}
\end{aligned}
$$

where $p_{t, n}(x, y, z)$ is the total measured sound pressure at the microphone position at the hologram plane and $p_{f w, n}\left(x_{n}, y_{n}, z_{n} \mid Z_{A}\right)$ is the forward model sound pressure in Eq. (17).

Since the surface impedance $Z_{A}$ is estimated using the finite surface method, Thomasson's alternative absorption coefficient, shown in Sec. II B 4, is calculated using Eq. (25). The cost functions are minimized using simplex optimization, ${ }^{34}$ by the use of the MATLAB function fminsearch. Here, the initial guesses for the method are the mean incident sound pressure amplitude over all reconstruction positions and the surface impedance estimated from an equivalent fluid model, Miki model, ${ }^{35}$ by using the radiation impedance for finite absorbers.

\section{MEASUREMENT SETUP}

All in situ measurements are performed in a large anechoic chamber at the Technical University of Denmark (DTU) with a volume of about $1000 \mathrm{~m}^{3}$ and a low frequency limit of about $50 \mathrm{~Hz} .{ }^{36}$ The absorber used in all measurements is the "Industrial" absorber from Rockfon, ${ }^{37}$ which has a thickness of $h_{d}=5 \mathrm{~cm}$ and a flow resistivity of $1.42 \times 10^{4} \mathrm{Ns} / \mathrm{m}^{4}$, measured with the impedance tube method described by Ren and Jacobsen. ${ }^{38}$

To approximate the assumption of a rigid infinite baffle (see Sec. II B), a large and heavy squared wooden plate of $3 \mathrm{~cm}$ thickness is placed in the anechoic chamber at DTU covering an area of about $14 \mathrm{~m}^{2}$ on which the absorber is centered (see Fig. 2). For the temporal subtraction method and the microphone array method, two different square absorber sizes are used with a side length of $1.2 \mathrm{~m}$ and $3.6 \mathrm{~m}$, respectively. For the finite surface method, only the sample with a side length of $1.2 \mathrm{~m}$ is used because the sample needs to be much smaller than the rigid baffle to fulfill the requirement of Eq. (17), where a rigid and infinite baffle with surface admittance of $\beta_{0}=0$ is considered.

The impulse response measurements, as well as the microphone array measurements are performed by placing a sound source (60-mm diameter two-way "coincidence source" loudspeaker unit produced by KEF mounted in a rigid plastic sphere $\left.{ }^{39}\right)$ at incident angles $\theta=\left\{0^{\circ}, 45^{\circ}, 60^{\circ}, 85^{\circ}\right\}$ above the absorber. The loudspeaker was adjusted such that the sound waves were always radiated from on-axis with respect to the center of the absorber. Since the measurements were performed on-axis with a nearly omni directional source, the directivity of the loudspeaker can be neglected. The distance between the center of the absorber and the source is kept constant at $2 \mathrm{~m}$ for all incidence angles. Plane wave radiation is assumed for $r>2 \lambda$ in the $1 / 3$ octave bands from $400 \mathrm{~Hz}$. Below this, spherical waves become more dominant and the theory of the finite surface method in Sec. II B is no longer valid. ${ }^{40,41}$ 
For impulse response measurements, the microphone is moved by the negative incident angle, keeping the distance between microphone and the absorber center constant at $0.8 \mathrm{~m}$. For the array measurements, a planar array with 60 pressure microphones is placed at horizontal $x-y$ planes above the absorber independent of the angle of incidence. The measurements are performed for three different planes, $z_{h 1}=4 \mathrm{~cm}, z_{h 2}=8 \mathrm{~cm}$, and $z_{h 3}=6 \mathrm{~cm}$; see Fig. 2 . For the SONAH reconstruction, just the two planes $z_{h 1}, z_{h 2}$ and the reconstruction plane $z_{s}=1 \mathrm{~cm}$ are used.

To minimize the positioning error of the microphone array during the measurements, a number of 60 measurement positions in 3 different hologram planes (180 points in total) are taken into account. The estimation of the sound pressure amplitude is based on the 24 center reconstruction positions. Only the center microphones are chosen because the error of the SONAH reconstruction increases at the edges of the array, as shown in Refs. 28 and 29.

For impulse response measurements a Brüel and Kjær (Nærum, Denmark) (B\&K) type 4189 microphone and the B\&K software DIRAC was used. A maximum-lengthsequence (MLS) signal with sampling frequency of $48 \mathrm{kHz}$, response length of $10 \mathrm{~s}$, and three averaging steps were applied. For microphone array measurements, a $10 \times 6$ microphone grid array, with B\&K microphone type 4957, and the B\&K PULSE analyzer were used. A pseudo-random noise sequence with sampling frequency of $48 \mathrm{kHz}$ was used as source signal. The spectral estimates were calculated with a frequency resolution of $1 \mathrm{~Hz}$ and bandwidth of $3200 \mathrm{~Hz}$ using a Hanning window with $70 \%$ overlap and 50 averages.

To validate the experiments, the resulting absorption coefficients of the different measurement methods are compared to the absorption coefficient measured with an impedance tube at normal incidence. The impedance tube used was a rectangular tube with a side length of $7 \mathrm{~cm}$ and microphone spacings of $4 \mathrm{~cm}$ and $16 \mathrm{~cm}$ to cover a broad frequency range. According to these dimensions, the tube gives sufficient accurate results between about $100 \mathrm{~Hz}$ and $2.3 \mathrm{kHz}$.

\section{EQUIVALENT FLUID MODEL}

To compare the measurements with theory, an equivalent fluid model is used. The model used in this study is the Miki model. ${ }^{35}$ This model shows an improvement of the well accepted Delany and Bazley model, ${ }^{42}$ including causality and minimum phase. It is based on one single material parameter, the flow resistivity $\sigma$. The normalized surface impedance $Z_{A}$ at oblique incidence $\theta$ for an absorber with thickness $h_{d}$ on a rigid infinite baffle is then calculated using the plane wave assumption, ${ }^{13}$

$$
Z_{A}=-\mathrm{j} Z_{0} \frac{k_{a}}{k_{z}(\theta)} \cot \left(k_{z}(\theta) h_{d}\right),
$$

where $k_{z}(\theta)=\sqrt{k_{a}^{2}-k_{0}^{2} \sin (\theta)^{2}}$ is the wave number perpendicular to the absorber, $Z_{0}$ is the characteristic impedance from Ref. 35, and $k_{a}$ is the wave number inside the porous material from Ref. 35. The equivalent fluid model is used for comparisons with measurements for all angles of incidence using different radiation impedances $Z_{F}$. Besides the radiation impedance based on an infinite sample size, $Z_{F}=1 / \cos (\theta)$, the radiation impedance based on a finite sample size, Eq. (20), is used. The corresponding absorption coefficient is either calculated with Eq. (25) for the finite sample, $\alpha^{\prime}(\theta)$, or with Eq. (3) for the infinite sample, $\alpha_{\infty}(\theta)$.

\section{RESULTS AND ANALYSIS}

\section{A. Pressure distribution on a finite absorber sample}

First, a simulation study showing the sound pressure distribution on the absorber surface and the rigid infinite baffle is presented. The equivalent fluid model, described in Sec. V, is used to calculate the surface impedance, $Z_{A}$. The absorber with a side length of $1.2 \mathrm{~m}$ is centered at the origin in one plane on a rigid infinite baffle. The sound pressure distribution at $z=0$ is calculated by using Eq. (19). The results are shown in Figs. 3 and 4, for $k e=\{4,16\}$, here, for $e=1.2 \mathrm{~m}$, corresponding to frequencies $f=\{182 \mathrm{~Hz}, 728 \mathrm{~Hz}\}$, and incident angles $\theta=\left\{0^{\circ}, 45^{\circ}, 60^{\circ}, 90^{\circ}\right\}$.

Considering the plane wave assumptions and normal sound incidence, the resulting surface impedance for approximating the absorber with the infinite absorber theory for $f$ $=182 \mathrm{~Hz}$ is $Z_{A}=0.80-\mathrm{j} 4.50$. The corresponding absorption coefficient, calculated by Eq. (3), is $\alpha=0.14$. The sound pressure on an infinite surface consists of two parts, the incident sound pressure and the sound pressure reflected from the surface. On the plane at $z=0$, the resulting sound pressure equals $1.86\left|p_{i}\right|$. In the same way, for $f=728 \mathrm{~Hz}, Z_{A}$ $=0.77-\mathrm{j} 0.89$ and $\alpha=0.78$. The resulting sound pressure on the plane at $z=0$ equals $1.21\left|p_{i}\right|$. Comparing the sound pressure calculated on an infinite sample with the sound pressure distribution on a finite sample for normal sound incidence, displayed in the upper left-hand side of Figs. 3 and 4, a fair agreement is found. The pressure at the center of the absorber equals $2.16\left|p_{i}\right|$ for $k e=4$ with a mean of $\bar{p}$ $=2.06\left|p_{i}\right|$ and a standard deviation of $\sigma_{p}=0.06\left|p_{i}\right|$ over the absorber surface. For $k e=16$, the sound pressure in the center equals $1.21\left|p_{i}\right|$ with $\bar{p}=1.20\left|p_{i}\right|$ and $\sigma_{p}=0.14\left|p_{i}\right|$. This implies that for small incidence angles, the infinite sample size assumption agrees reasonably well with the finite sample size assumption.

For incidence angles of $45^{\circ}$ and $60^{\circ}$, the sound pressure of an infinite absorber with $f=182 \mathrm{~Hz}$ equals $1.81\left|p_{i}\right|$ and $1.75\left|p_{i}\right|$, respectively. With $f=728 \mathrm{~Hz}$, the sound pressure equals $1.20\left|p_{i}\right|$ and $1.26\left|p_{i}\right|$ for incidence angles of $45^{\circ}$ and $60^{\circ}$, respectively. Comparing these calculations with the sound pressure distribution of a finite sample, shown in Figs. 3 and 4, large deviations are found. For an incidence angle of $60^{\circ}$, the pressure in the center of the absorber equals $1.72\left|p_{i}\right|$ for $k e=4$ (corresponding to a frequency of $182 \mathrm{~Hz}$ ) with a mean of $\bar{p}=1.73\left|p_{i}\right|$ and a standard deviation of $\sigma_{p}=0.40\left|p_{i}\right|$ over the absorber surface. For $k e=16$ (corresponding to a frequency of $728 \mathrm{~Hz}$ ), the sound pressure in the center equals 3.81|p $p_{i} \mid$ with $\bar{p}=2.51\left|p_{i}\right|$ and $\sigma_{p}=1.16\left|p_{i}\right|$. Furthermore, a more complicated pattern of the sound pressure on the finite absorber and the surrounding is found. For an incidence angle of $45^{\circ}$ and $k e=4$, the sound 


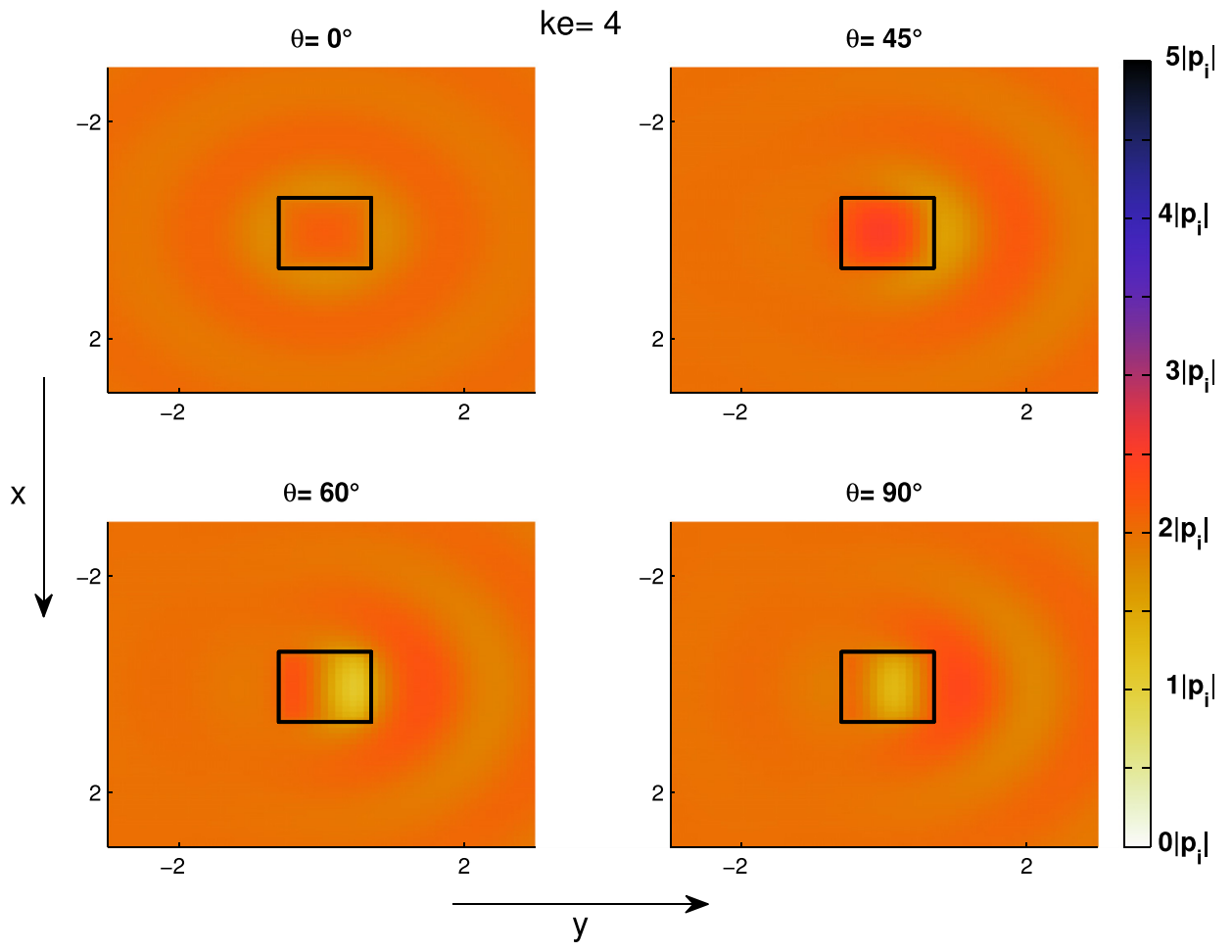

FIG. 3. (Color online) Simulated sound pressure distribution (magnitude) over a finite absorber with a side length of $1.2 \mathrm{~m}$ for $k e=4$, here, $f=182 \mathrm{~Hz}$, and different incident angles $\theta=\left\{0^{\circ}, 45^{\circ}, 60^{\circ}, 90^{\circ}\right\}$. The rectangle indicates the dimensions of the absorbing material.

pressure is increased up to $2.5\left|p_{i}\right|$. This shows that there is a pressure increase due to scattering from the absorber edges. For an incidence angle of $60^{\circ}$ and $k e=4$, a pressure minimum on the right-hand side of the absorber is found, indicating a high absorption. Figure 4 shows that for $k e=16$, the pressure decreases and increases occur over the entire absorber surface and the surrounding boundary with pressure increases up to $4.6\left|p_{i}\right|$.

Assuming grazing incidence $\left(\theta=90^{\circ}\right)$ on an infinitely large absorber sample, the sound pressure equals $2\left|p_{i}\right|$ for all ke. In Figs. 3 and 4, it is found that, by assuming a finite sample size, there are pressure increases and decreases on the absorber and the surrounding boundary (this shows that at grazing incidence, sound power is absorbed). This indicates that the finiteness of the absorber has a significant influence on the pressure field and, consequently, on the absorption coefficient.

Since the calculation of the absorption coefficient using the temporal subtraction method and the microphone array method are based on an infinite absorber sample, they cannot account for the precise sound pressure distribution over the absorber and the surrounding, whereas the finite surface method can.

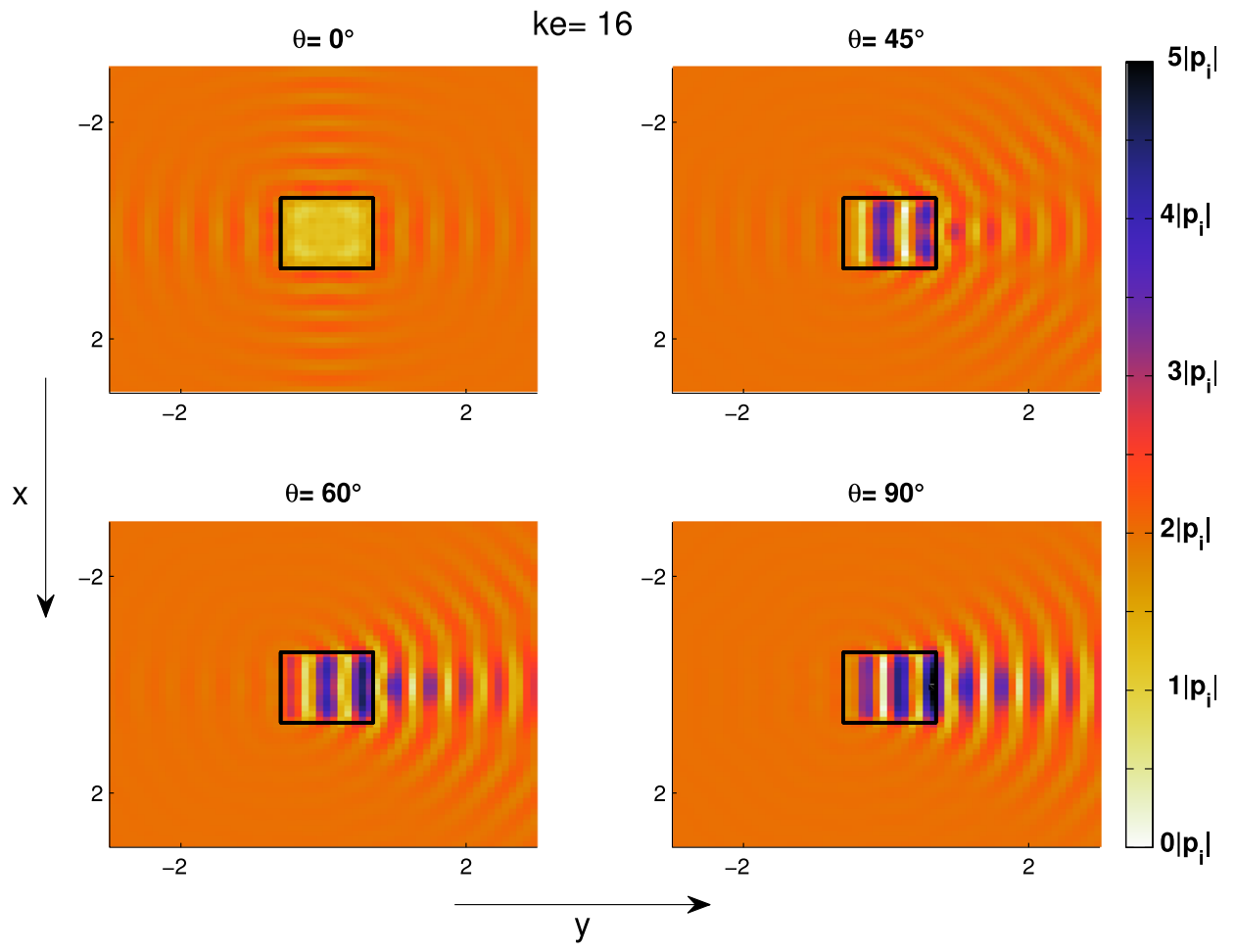

FIG. 4. (Color online) Simulated sound pressure distribution (magnitude) over a finite absorber with a side length of $1.2 \mathrm{~m}$ for $k e=16$, here, $f$ $=728 \mathrm{~Hz}$ and different incident angles $\theta=\left\{0^{\circ}, 45^{\circ}, 60^{\circ}, 90^{\circ}\right\}$. The rectangle indicates the dimensions of the absorbing material. 


\section{B. Measurement results}

In this section, the estimated absorption coefficients from in situ measurements are shown and compared to the equivalent fluid model using different radiation impedances. The results are given in Figs. 5 and 6, with incidence angles of $\theta=\left\{0^{\circ}, 45^{\circ}, 60^{\circ}, 85^{\circ}\right\}$ and absorber side length of $1.2 \mathrm{~m}$ and $3.6 \mathrm{~m}$. For the theory of the finite surface method to hold, the size of the absorber under test needs to be much smaller than the rigid baffle, especially for large incidence angles. Therefore, results of the sample with side length of $3.6 \mathrm{~m}$ are excluded for large incidence angles. Each figure contains measurements using different methods, as well as simulated results. The results are shown in $1 / 3$ octave bands between $200 \mathrm{~Hz}$ and $2500 \mathrm{~Hz}$. In the following, the results are described and explained more comprehensively.

\section{Equivalent fluid model}

Figure 5(a) shows good agreement between the impedance-tube measurement and the equivalent fluid model at normal incidence for the entire frequency range under test for an absorber with side length of $1.2 \mathrm{~m}$.

For the absorbers with a side length of $3.6 \mathrm{~m}$ and normal sound incidence, shown in Fig. 5(b), the absorption coefficient computed using the radiation impedance of a finite
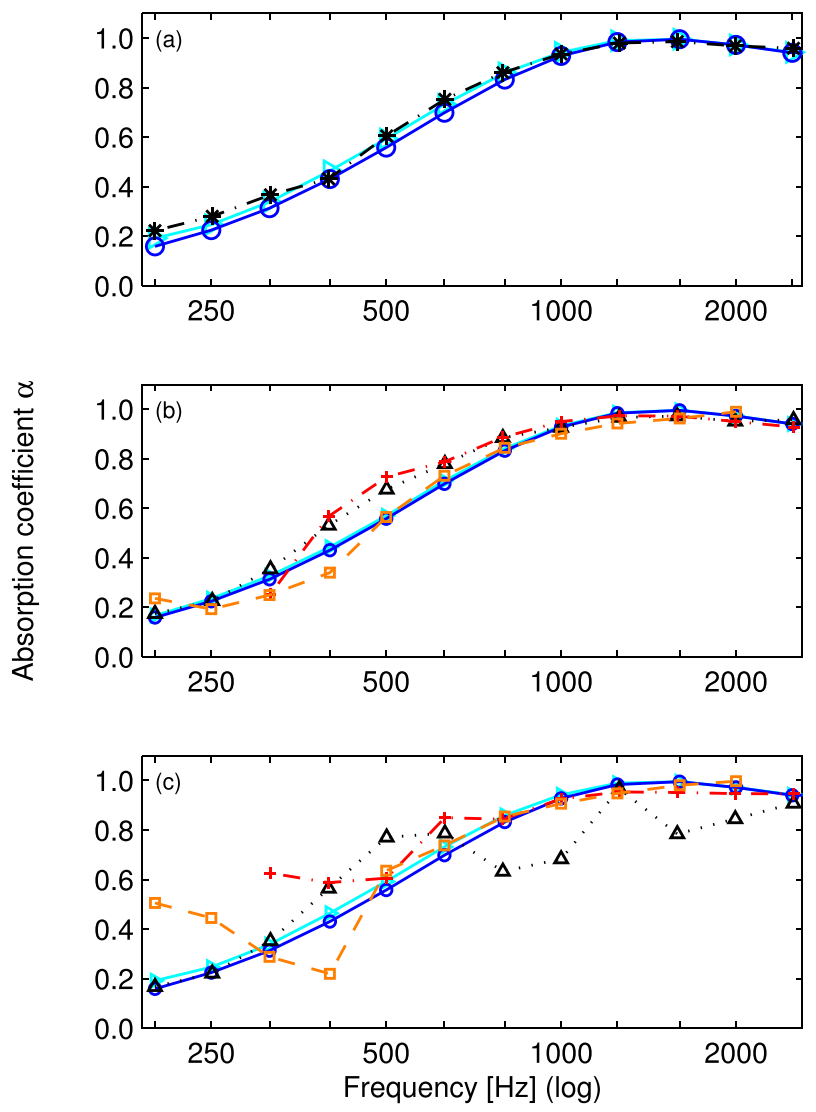

FIG. 5. (Color online) Absorption coefficient of a finite absorber sample at normal sound incidence, $\theta=0^{\circ}$. (- $\left.\bigcirc\right)$ Miki model: Radiation impedance based on an infinite sample size, $(-\triangleright)$ Miki model: Radiation impedance based on a finite sample size, $(\cdots \Delta)$ Temporal subtraction method, $(-$. $-+)$ microphone array method, $(-\cdot-*)$ impedance tube, $(--\square)$ finite surface method. (a) Impedance tube measurement, (b) absorber with a side length of $3.6 \mathrm{~m}$, and (c) absorber with a side length of $1.2 \mathrm{~m}$.
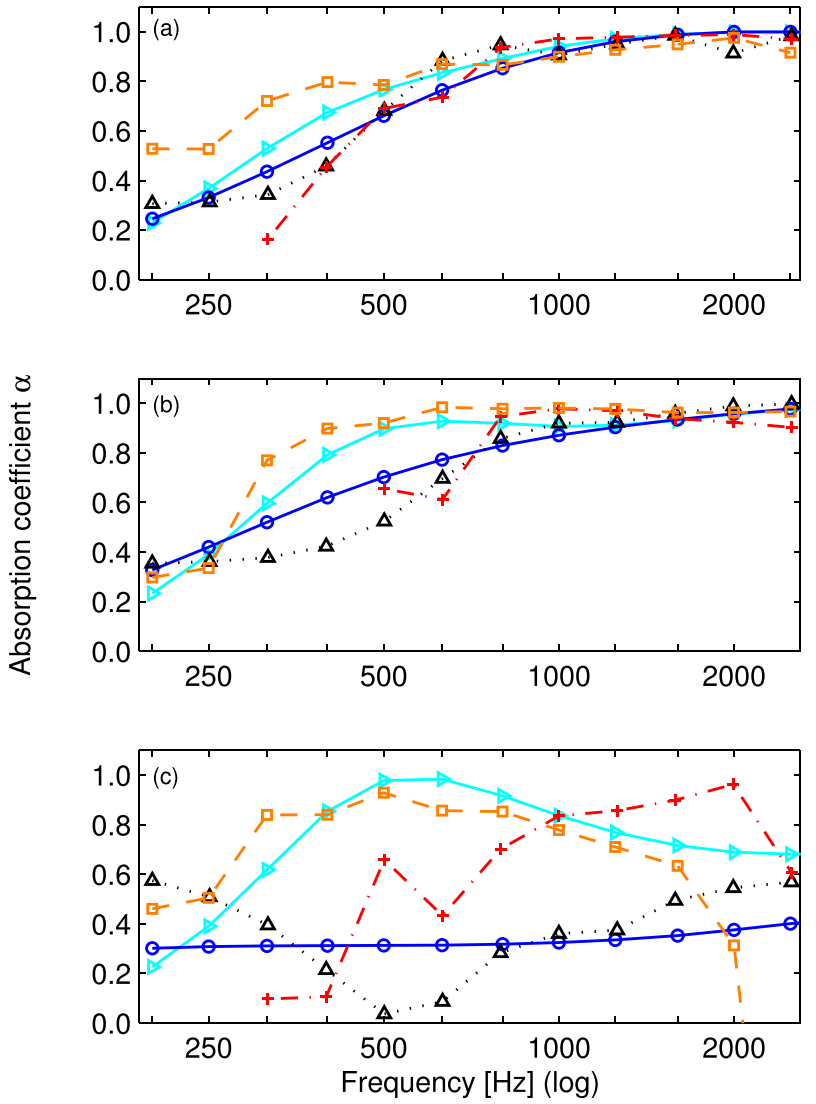

FIG. 6. (Color online) Absorption coefficient of a sample with a side length of $1.2 \mathrm{~m}$ and different incident angles. (- $\bigcirc)$ Miki model: Radiation impedance based on an infinite sample size, $(-\triangleright)$ Miki model: Radiation impedance based on a finite sample size, $(\cdots \Delta)$ temporal subtraction method, $(-\cdot-+)$ microphone array method, $(--\square)$ finite surface method. (a) $\theta=45^{\circ}$, (b) $\theta=60^{\circ}$, and (c) $\theta=85^{\circ}$.

sample, Eq. (20), agrees well with the one using the radiation impedance of an infinite sample, $Z_{F}=1 / \cos \theta$. For the absorber with a side length of $1.2 \mathrm{~m}$ and normal sound incidence, shown in Fig. 5(c), the equivalent fluid model using the radiation impedance of a finite sample and the radiation impedance of an infinite sample differ slightly for frequencies below $1 \mathrm{kHz}$.

As seen in Fig. 6 (for the absorber with a side length of $1.2 \mathrm{~m}$ ) with increasing incidence angle, the calculated absorption coefficient assuming an infinitely large absorber sample becomes small and approaches zero at grazing incidence. The absorption coefficient based on the radiation impedance of a finite sample, however, still shows a high absorption, especially in the medium frequency range. This behavior reflects the observations from the simulated sound pressure distribution on the absorber, as shown in Sec. VI A.

\section{Validation of the subtraction and microphone array method}

To indicate the validity of the measurement methods used in the experiments, the absorber sample with a side length of $3.6 \mathrm{~m}$ placed on a large and heavy wooden plate is used. Figure 5(b) shows that both in situ methods, the temporal subtraction method and the microphone array method, have good agreement with the equivalent fluid model 
between $315 \mathrm{~Hz}$ and $2.5 \mathrm{kHz}$, approximately. Thus, both measurement methods are valid for further measurements and comparisons.

\section{The temporal subtraction and the microphone array methods}

Figures 5(b) and 5(c) and 6(a)-6(c) also show the estimated absorption coefficients using the temporal subtraction method. Measurement results not shown in this paper indicate that good results are obtained by using the absorber sample with a side length of $3.6 \mathrm{~m}$ for incident angles up to $60^{\circ}$, approximately. These observations are similar to the results in Ref. 7 that for larger incident angles more advanced methods should be considered to account for the diffracted wave fronts. By using a small absorber sample with a side length of $1.2 \mathrm{~m}$, it is difficult to get sufficiently accurate results over the entire frequency range, even for small incident angles, as shown in Fig. 5(c). Even though a very detailed post-processing method is used, the time difference between the directly reflected part and the diffracted reflected part of the impulse response becomes too small to obtain accurate results. These observations agree well with the results from Sec. VI A, showing that the sound pressure distribution over and around a small absorber sample becomes very complicated. This effect becomes more dominant with increasing incidence angle, which implies that for finite and small absorber samples one needs a more advanced method that accounts for the influence of the absorber edges on the absorption coefficient.

With the microphone array method, similar observations are found. As shown in Fig. 5(b), good agreement between the results of this method and the equivalent fluid models is found for frequencies above $630 \mathrm{~Hz}$ with an absorber with a side length of $3.6 \mathrm{~m}$. At low frequencies, the error from SONAH increases slightly, which yields a deviation from the equivalent fluid model.

As seen in Fig. 5(c) for the absorber with a side length of $1.2 \mathrm{~m}$, the microphone array method agrees well with the equivalent fluid models for frequencies above $500 \mathrm{~Hz}$. With increasing incidence angle, the results of the microphone array method are very similar to the observations from the temporal subtraction method. That can be seen in Figs. 6(a) and 6(b). Near gracing incidence, no agreement with the theory is found. In the low frequency range, greater deviations between the measurement results and theory are found for all incident angles.

At low frequencies, the spatial sampling of the array is just a small fraction of the wavelength. Therefore, the dimensions of the array, the plane wave limitation in Sec. $\mathrm{IV}$, and the distance between the measurement planes can limit the accuracy of the results in the low frequency range. In the presented measurement setup, the distance between the two measurement planes is as small as $4 \mathrm{~cm}$. Therefore, the phase differences between two consecutive microphones become negligible. Hence, the reflection factor approaches one based on the theory described in Sec. II A, which yields an absorption coefficient of zero. This results from the fact that the effective properties of the array change with the angle of incidence. ${ }^{28}$ As shown in Sec. IV, the plane wave assumption from Sec. II B is no longer valid for the $1 / 3$ octave bands below $400 \mathrm{~Hz}$. Since the wave fronts below $400 \mathrm{~Hz}$ are assumed to be spherical, the measured sound pressure consists of a range of incidence angles. Therefore, the reflection factor cannot be calculated as a spatial averaging with Eq. (30) for low frequencies. Furthermore, assuming spherical wave incidence on the absorber surface, the surface impedance changes over the material surface. ${ }^{43}$ Due to this limitations in the low frequency range, the microphone array results in Figs. 5, 6(a), and 6(c) are only reported for the $1 / 3$ octave bands from $315 \mathrm{~Hz}$. For an incidence angle of $60^{\circ}$, negative results occurred even for frequencies below $500 \mathrm{~Hz}$. In further studies, another setup using a larger distance between the measurement planes could extend the valid frequency range.

It should be noted that at low frequencies the edge effect becomes significant. In the present measurement setup, the distance between the source and the absorber is kept constant as $2 \mathrm{~m}$. In order to obtain a better low frequency estimate, the source could be placed closer to the sample to reduce the edge effect and better emulate an infinite absorber. ${ }^{33}$ Nevertheless, another interesting alternative would be to use the array data to reconstruct the surface impedance $Z_{A}$, from which the absorption coefficient or other material properties could be estimated (as done in the finite surface method, see Sec. III C).

It is found that the temporal subtraction and microphone array methods give good results for small incident angles using a finite sample. For $\theta=45^{\circ}$, e.g., both methods agree well with the equivalent fluid model using the radiation impedance of an infinite sample.

\section{The finite surface method}

The real and imaginary part of the estimated surface impedance, calculated with Eq. (32), are shown in Fig. 7 for incident angles $\theta=\left\{0^{\circ}, 45^{\circ}, 60^{\circ}, 85^{\circ}\right\}$ and an absorber with a side length of $1.2 \mathrm{~m}$. The corresponding estimated absorption coefficient is shown in Figs. 5(b), 5(c), and 6(a)-6(c).

From Figs. 7(a)-7(c), it is found that for incidence angles below $60^{\circ}$ the estimated imaginary part of the surface impedance agrees well with the equivalent fluid model over the entire frequency range. Besides this, the estimated real part of the surface impedance differs from the equivalent fluid model. This can, e.g., be seen in Figs. 7(b) and 7(c) for incidence angles of $45^{\circ}$ and $60^{\circ}$.

For an incidence angle of $85^{\circ}$, shown in Fig. 7(d), the estimated real part of the surface impedance shows good agreement with theory for frequencies between $315 \mathrm{~Hz}$ and $1.5 \mathrm{kHz}$. The imaginary part shows good agreement with the equivalent fluid model for frequencies between $500 \mathrm{~Hz}$ and $1.5 \mathrm{kHz}$.

In the presented study, the initial guesses for $Z_{A}$ in Eq. (32) are the surface impedances of the equivalent fluid model. To reduce computational time and gain good results, the initial guess should be as close as possible to the true value. In practical measurement situations, the flow 


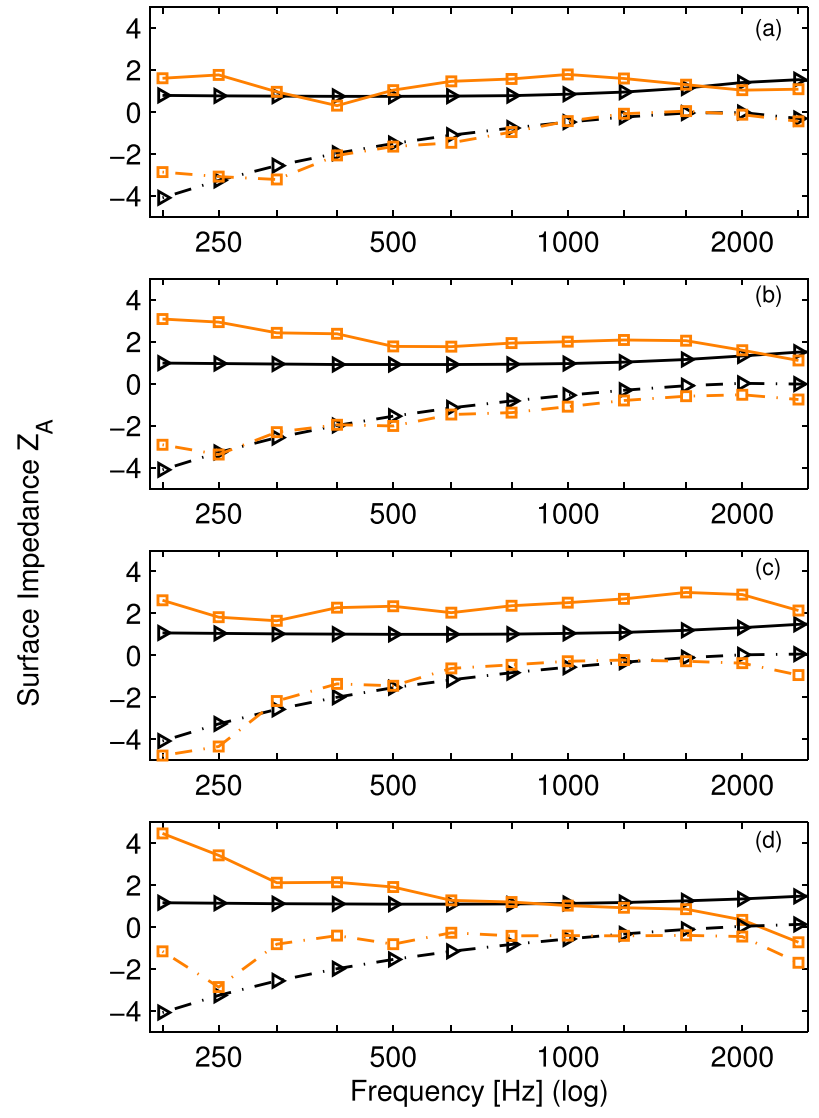

FIG. 7. (Color online) Normalized surface acoustic impedance of a sample with side length of $1.2 \mathrm{~m}$ and different incident angles. ( $\square$ ) Real part-finite surface method, $(--\square)$ imaginary part-finite surface method, $(-\triangleright)$ real part-Miki model, $(--\triangleright)$ imaginary part-Miki model. (a) $\theta=0^{\circ}$, (b) $\theta=45^{\circ}$, (c) $\theta=60^{\circ}$, and (d) $\theta=85^{\circ}$.

resistivity of the material under test is not always known. Thus, besides the calculated value from the equivalent fluid model, the results of an impedance tube measurement at normal sound incidence could be used as an initial guess. Furthermore, a more advanced optimization, such as the Bayesian inversion, could be used. With such an approach, prior information about the unknown parameters can be taken into account.

Below the incidence angle of $60^{\circ}$, the resulting absorption coefficient of the finite surface method agrees well with theory for frequencies above $500 \mathrm{~Hz}$. Below this frequency, larger deviations from the theory are found. Besides the spatial error, explained in Sec. VIB 3, there exists a computational bias in the reconstructed sound pressure due to SONAH. ${ }^{26}$ This bias can be assumed to be almost constant for all considered frequencies. Since the influence of the bias is directly related to the absorption coefficient, the error in the results is more dominant for low frequencies where the absorption is low, than that for high frequencies where the absorption is high. As shown in Sec. IV, the plane wave assumption from Sec. IIB is no longer valid for the $1 / 3$ octave bands below $400 \mathrm{~Hz}$. Therefore, the results from the finite surface method are only reliable for the $1 / 3$ octave bands starting from $400 \mathrm{~Hz}$.

Especially near grazing incidence $\left(\theta=85^{\circ}\right)$, in Fig. 6(c), the finite surface method gives a promising agreement with the equivalent fluid model by using the finite surface radiation impedance between $400 \mathrm{~Hz}$ and $1.5 \mathrm{kHz}$, approximately.

The finite surface method shows good agreement with theory, especially for large incidence angles. Nevertheless, in the low frequency range some uncertainties in the method are observed. Besides a bias in the reconstructed pressure due to SONAH and the finite measurement aperture, the method is limited due to plane wave assumption shown in Sec. IV.

Considering the abovementioned limitations of the method, we expect more accurate results by carefully choosing a better microphone grid with greater distances between the hologram planes, a greater distance between source and receiver, and advanced post processing in further investigations.

The results show that the assumption of simple plane wave reflections from an infinite absorber, shown in Sec. II A, can be justified when computing the absorption coefficient for the large sample with a side length of $3.6 \mathrm{~m}$ for incidence angles below $60^{\circ}$ in situ. For the small sample with side length of $1.2 \mathrm{~m}$, its performance was degraded even for small incidence angles. Thus, Thomasson's alternative absorption coefficient for finite absorbers in Sec. II B 4 is needed to compute the absorption coefficient in situ for incidence angles larger than $60^{\circ}$.

\section{CONCLUSIONS}

A method to measure the acoustic properties of sound absorbers at oblique sound incidence by taking the finiteness of the absorber into account are proposed, based on a sound field model for finite absorbers considering scattering reflections. Three different measurement methods are compared: the temporal subtraction method, the microphone array method, and the finite surface method. With the finite surface method, the finiteness of the absorber is taken into account in an inverse manner. For small absorber samples and large incidence angles, the influence of finiteness of the absorption coefficient is substantial. Simplified methods to calculate the absorption coefficient assuming an infinitely large absorber sample and plane wave incidence onto the surface show that no sound power is absorbed at grazing incidence, while in real measurement situations, sound is absorbed. Good agreement between the proposed finite surface method and the equivalent fluid model using the radiation impedance for finite absorbers is found, especially for large incidence angles and small specimens.

${ }^{1}$ ISO 10534-2:1998, "Acoustics-Determination of sound absorption coefficient and impedance in impedance tubes-Part 2: Transfer-function method" (International Organization for Standardization, Geneva, 1998).

${ }^{2}$ ISO 354:2003, "Acoustics-Measurement of sound absorption in a reverberation room" (International Organization for Standardization, Geneva, 2003).

${ }^{3}$ S.-I. Thomasson, "On the absorption coefficient," Acta Acust. Acust. 44(4), 265-273 (1980).

${ }^{4}$ S.-I. Thomasson, "Theory and experiments on the sound absorption as function of the area," Report No. TRITA-TAK-8201, KTH Stockholm (1982).

${ }^{5}$ C.-H. Jeong and J. Brunskog, "The equivalent incidence angle for porous absorbers backed by a hard surface," J. Acoust. Soc. Am. 134(6), 4590-4598 (2013). 
${ }^{6} \mathrm{U}$. Ingard and R.-H. Bolt, “A free field method of measuring the absorption coefficient of acoustic materials," J. Acoust. Soc. Am. 23(5), 509-516 (1951).

${ }^{7}$ E. Mommertz, "Angle-dependent in-situ measurements of reflection coefficients using a subtraction technique," Appl. Acoust. 46, 251-263 (1995).

${ }^{8}$ P. Robinson and N. Xiang, "On the subtraction method for in-situ reflection and diffusion coefficient measurements," J. Acoust. Soc. Am. 127(3), EL99-EL104 (2010).

${ }^{9}$ K. Hirosawa, K. Takashima, H. Nakagawa, M. Kon, A. Yamamoto, and W. Lauriks, "Comparison of three measurement techniques for the normal absorption coefficient of sound absorbing materials in the free field," J. Acoust. Soc. Am. 126(6), 3020-3027 (2009).

${ }^{10}$ A. Yori and M. Moeser, "A measurement method for the sound absorption coefficient for arbitrary sound fields and surfaces," Acta Acust. Acust. 101(4), 668-674 (2015).

${ }^{11}$ E. Brandao, A. Lenzi, and S. Paul, "A review on in situ impedance and sound absorption measurement techniques," Acta Acust. Acust. 101, 443-463 (2015).

${ }^{12}$ F.-P. Mechel, "On sound absorption of finite-size absorbers in relation to other radiation impedances," J. Sound Vib. 135(2), 225-262 (1989).

${ }^{13} \mathrm{~J} .-\mathrm{S}$. Pyett, "The acoustic impedance of a porous layer at oblique incidence," Acta Acoust. 3, 375-382 (1953).

${ }^{14}$ F.-P. Mechel, Schallabsorber-Band 1, Äußere Schallfelder, Wechselwirkungen (Sound absorbers-Part 1, external sound fields, interactions) (Hirzel, Stuttgart, 1989), pp. 39-51, 232-276.

${ }^{15}$ C.-L. Morfey, Dictionary of Acoustics (Academic, San Diego, CA, 2001), p. 315.

${ }^{16}$ S.-I. Thomasson, "Reflection of waves from a point source by an impedance boundary," J. Acoust. Soc. Am. 59, 780-785 (1976).

${ }^{17}$ P.-M. Morse and K.-U. Ingaard, Theoretical Acoustics (Princeton University Press, Princeton, NJ, 1968), pp. 155-163.

${ }^{18} \mathrm{~J}$. Brunskog, "Forced sound transmission of finite single leaf walls using a variational technique,” J. Acoust. Soc. Am. 132(3), 1482-1493 (2012).

${ }^{19}$ D. Holmberg, P. Hammer, and E. Nilsson, "Absorption and radiation impedance of finite absorbing patches," Acta Acust. Acust. 89, 406-415 (2003)

${ }^{20}$ J. Brunskog and P. Hammer, "Rigid indenter excitation of plates," Acta Acust. Acust. 89, 460-470 (2003).

${ }^{21}$ W.-L. Li and H.-J. Gibeling, "Determination of the mutual radiation resistances of a rectangular plate and their impact on the radiated sound power," J. Sound Vib. 229(5), 1213-1233 (2000).

${ }^{22}$ J.-L. Davy, D.-J. Larner, R.-R. Wareing, and J.-R. Pearse, "The average specific forced radiation wave impedance of a finite rectangular panel," J. Acoustc. Soc. Am. 136, 525-536 (2014).

${ }^{23}$ M. Nolan, C.-H. Jeong, J. Brunskog, J. Rodenas, F. Chevillotte, and L. Jaousen, "Different radiation impedance models for finite porous absorbers," in Proceedings of EuroNoise 2015 (2015).

${ }^{24}$ ISO 13472-1:2012, "Acoustics-Measurement of sound absorption properties of road surfaces in situ-Part 1: Extended surface method" (International Organization for Standardization, Geneva, 2012).
${ }^{25}$ J.-D. Maynard, E.-G. Williams, and Y. Lee, "Nearfield acoustic holography: Theory of generalized holography and the development of NAH," J. Acoust. Soc. Am. 78(4), 1395-1413 (1985).

${ }^{26} \mathrm{~J}$. Hald, "Basic theory and properties of statistically optimized near-field acoustical holography," J. Acoust. Soc. Am. 125, 2105-2120 (2009).

${ }^{27}$ E. Fernandez-Grande and F. Jacobsen, "Sound field separation with a double layer velocity transducer array," J. Acoust. Soc. Am. 130(1), 5-8 (2011).

${ }^{28}$ E. Fernandez-Grande, F. Jacobsen, and Q. Leclere, "Sound field separation with sound pressure and particle velocity measurements," J. Acoust. Soc. Am. 132(6), 3818-3825 (2012)

${ }^{29}$ E. Fernandez-Grande, "Near-field acoustic holography with sound pressure and particle velocity measurements," Ph.D. thesis, Technical University of Denmark (DTU), 2012.

${ }^{30}$ P.-C. Hansen, Analysis of Discrete Ill-Posed Problems (SIAM, Philadelphia, 1992).

${ }^{31}$ P.-C. Hansen, Range-Deficient and Discrete Ill-Posed Problems by Means of the L-Curve (SIAM, Philadelphia, 1998).

${ }^{32}$ M. Tamura, "Spatial Fourier transform method of measuring reflection coefficients at oblique incidence. I: Theory and numerical examples," J. Acoust. Soc. Am. 88, 2259-2264 (1990).

${ }^{33}$ M. Tamura, "Spatial Fourier-transform method for measuring reflection coefficients at oblique incidence. II. Experimental results," J. Acoust. Soc. Am. 97, 2255-2262 (1995).

${ }^{34}$ J.-A. Nelder and R. Mead, "A simplex-method for function minimization," Comput. J. 7(4), 308-313 (1965).

${ }^{35}$ Y. Miki, "Acoustical properties of porous materials-Modification of Delany-Bazley models," J. Acoust. Soc. Jpn. 11, 19-24 (1990).

${ }^{36}$ F. Ingerslev, P.-K. Müller, and J. Kristensen, "The anechoic chambers at the Technical University of Denmark," Brüel and Kjær Technical Note (1968), Vol. 2.

${ }^{37}$ Rockfon, "Rockfon Industrial Opal/Black/Nature," Product catalog from Rockfon, available at http://products.exp-en.rockfon.com/media/286660/ datasheet_exp_industrial_02.2011.pdf (Last viewed 2/13/2015).

${ }^{38} \mathrm{M}$. Ren and F. Jacobsen, "A method of measuring the dynamic flow resistance and reactance of porous materials," Appl. Acoust. 39(4), 265-276 (1993).

${ }^{39}$ L.-R. Fincham, A. Jones, and R.-H. Small, "The influence of room acoustics on reproduced sound, Part 2: Design of wideband coincident-source loudspeakers," Audio Eng. Soc. 87, 2877 (1989).

${ }^{40} \mathrm{U}$. Ingard, "On the reflection of a spherical sound wave from an infinite plane,” J. Acoust. Soc. Am. 23, 329-335 (1951).

${ }^{41}$ C.-H. Jeong, "Absorption and impedance boundary conditions for phased geometrical acoustic methods," J. Acoust. Soc. Am. 132, 2347-2358 (2012).

${ }^{42}$ M.-E. Delany and E.-N. Bazley, "Acoustical characteristics of fibrous absorbent materials," Appl. Acoust. 3, 105-116 (1970).

${ }^{43}$ R. Dragonetti and R.-A. Romano, "Considerations on the sound absorption of non locally reacting porous layers,” Appl. Acoust. 87, 46-56 (2015). 